\title{
Emergent Moments and Random Singlet Physics in a Majorana Spin Liquid
}

\author{
Sambuddha Sanyal $\odot,{ }^{1}$ Kedar Damle, ${ }^{2}$ J. T. Chalker $\odot,{ }^{3}$ and R. Moessner ${ }^{4}$ \\ ${ }^{1}$ Department of Physics, Indian Institute of Science Education and Research (IISER) Tirupati, Tirupati 517507, India \\ ${ }^{2}$ Department of Theoretical Physics, Tata Institute of Fundamental Research, Mumbai 400005, India \\ ${ }^{3}$ Theoretical Physics, University of Oxford, Parks Road, Oxford OX1 3PU, United Kingdom \\ ${ }^{4}$ Max Planck Institute for the Physics of Complex Systems, Nöthnitzer Straße 38, 01187 Dresden, Germany
}

(Received 25 December 2020; revised 12 June 2021; accepted 2 August 2021; published 13 September 2021)

\begin{abstract}
We exhibit an exactly solvable example of a SU(2) symmetric Majorana spin liquid phase, in which quenched disorder leads to random-singlet phenomenology of emergent magnetic moments. More precisely, we argue that a strong-disorder fixed point controls the low temperature susceptibility $\chi(T)$ of an exactly solvable $S=1 / 2$ model on the decorated honeycomb lattice with vacancy and/or bond disorder, leading to $\chi(T)=\mathcal{C} / T+\mathcal{D} T^{\alpha(T)-1}$, where $\alpha(T) \rightarrow 0$ slowly as the temperature $T \rightarrow 0$. The first term is a Curie tail that represents the emergent response of vacancy-induced spin textures spread over many unit cells: it is an intrinsic feature of the site-diluted system, rather than an extraneous effect arising from isolated free spins. The second term, common to both vacancy and bond disorder [with different $\alpha(T)$ in the two cases] is the response of a random singlet phase, familiar from random antiferromagnetic spin chains and the analogous regime in phosphorus-doped silicon (Si:P).
\end{abstract}

DOI: 10.1103/PhysRevLett.127.127201

Quantum spin liquids [1-3] are of interest as topological states of magnets. Specifically, their low-energy physics contains unusual degrees of freedom, such as emergent gauge fields and fractionalized excitations carrying their gauge charges [4]. As these need not correspond to known elementary particles, spin liquids can even make quasiparticles with unusual quantum numbers uniquely available [5]. In particular, these excitations can then exhibit interesting and unusual low-energy behavior of their own, where the bulk of the spin liquid can act as a matrix mediating emergent interactions between them. Although elusive in the ground state of a clean system, these excitations are arguably the most fundamental property of a spin liquid.

Disorder turns out to be a particularly revealing probe, as it can lead to the appearance ("nucleation") of such gaugecharged fractionalized degrees of freedom already at $T=0$. For spin liquids with gapless excitations, even weak disorder can redistribute the low-energy spectral weight [6-9]. In reverse, probing the low-energy physics of an experimental compound can provide insights into the amount and nature of disorder present in a particular material $[10,11]$. In addition, strong disorder can drive a

Published by the American Physical Society under the terms of the Creative Commons Attribution 4.0 International license. Further distribution of this work must maintain attribution to the author(s) and the published article's title, journal citation, and DOI. Open access publication funded by the Max Planck Society. quantum magnet to other unusual states, for instance, the random singlet state familiar from the physics of random antiferromagnetic spin chains studied in pioneering theoretical work [12,13] motivated by early experiments [14]. This state exhibits a random pattern of singlet bonds between moments, and a concomitant broad distribution of triplet excitation energies.

Crucially, our present understanding [15-22] of this physics in one dimension rests firmly on the twin pillars of an exact calculation for a special integrable model on the one hand [15,22-24], and Fisher's [15,16] asymptotically exact renormalization group ( $R G)$ analysis of the general case on the other hand. This RG analysis quantifies how the distribution of effective exchange couplings broadens without limit as one approaches the low-energy fixed point of the RG. Turning to higher dimensions, experimental results on Si:P [25-27] also admit a phenomenological random-singlet description. However, the physics of local moments in Si:P at still lower temperatures is expected to be different, since strong-disorder RG studies conclude that the renormalized disorder strength $[27,28]$ in the low-energy limit does not grow without bound in this three-dimensional case.

A somewhat different random-singlet phenomenology [29-31] has been recently employed to describe [32] several frustrated $\mathrm{S}=1 / 2$ antiferromagnets with quenched disorder [10,11,33-35]. This has a crucial additional ingredient $[30,32]$ with no analog in the original randomsinglet phenomenology of spin chains or Si:P, namely, the formation of emergent disorder-induced local moments at an intermediate energy; these ultimately freeze into a random singlet state at still lower energies. 
Here, we place these intriguing new ideas on a firm foundation with an exact calculation of such emergent moments and random-singlet behavior in the SU(2)invariant version [36] of Kitaev's [37] integrable model of a honeycomb-lattice spin liquid. Using arbitraryprecision numerics and analytical calculations to span the full range of low temperatures, we study the effects of vacancies (dilution by nonmagnetic impurities) and bond (exchange) disorder on the susceptibility, obtaining

$$
\chi(T)=\mathcal{C} / T+\mathcal{D} T^{\alpha(T)-1} .
$$

The Curie term $\mathcal{C} / T$ arises exclusively from vacancyinduced emergent local moments that fail to form singlets. These emergent moments are a cooperative effect involving a large number of spins, and represent unusual quantum analogs of vacancy-induced orphan spin textures in classical frustrated magnets [38-43]. The second term displays the characteristic divergence associated with a strong-disorder random singlet phase, and is common to vacancy and bond disorder [albeit with different $\alpha(T) \rightarrow 0$ as $T \rightarrow 0]$. In the diluted case, it displays an interesting crossover which we characterize fully.

The integrable model [36] studied here hosts a SU(2) symmetric Majorana spin liquid $[36,44,45]$ in which the $\mathbb{Z}_{2}$ fluxes are static and gapped. It has $S=1 / 2$ moments $\vec{S}_{R}$ on sites $R$ of a decorated honeycomb lattice in which each honeycomb site $\vec{r}$ is replaced by a triangle $\vec{r}$ consisting of three sites $R_{1 / 2 / 3}(\vec{r})$ (see Fig. 1). The three spins of a given triangle are coupled to each other with a large antiferromagnetic exchange $J$, the largest energy scale in the Hamiltonian. As a result, the low-energy physics is controlled by states in which each triangle is in one of two $S_{\text {tot }}=1 / 2$ doublet states. These two doublets are distinguished by the "orbital" quantum number $\tau_{\vec{r}}^{z}= \pm 1$. Neighboring triangles are coupled by a multispin interaction of strength $K$ that is sensitive to the "orbital wave function" of the total spin state of each triangle.

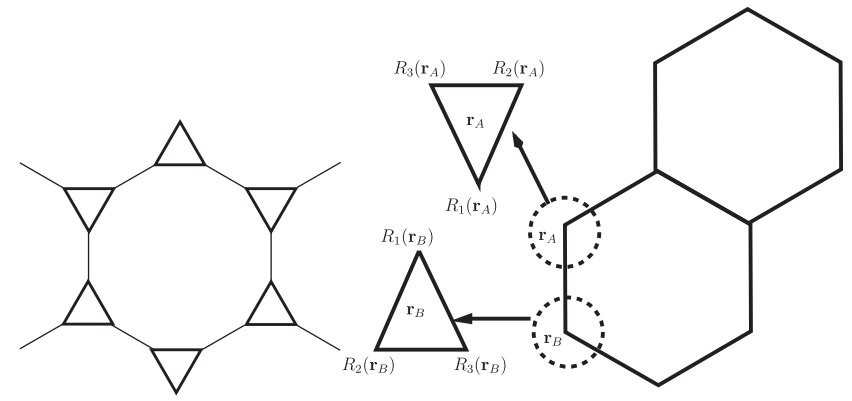

FIG. 1. The decorated honeycomb, or star, lattice (left) is obtained from the honeycomb on replacing every $A[B]$ sublattice site $\vec{r}_{A}\left[\vec{r}_{B}\right]$ of the honeycomb (right) by an up [down] pointing triangle made up of sites $R_{1 / 2 / 3}\left(\vec{r}_{A}\right)\left[R_{1 / 2 / 3}\left(\vec{r}_{B}\right)\right]$.
When $K \ll J$, the low-energy Hamiltonian can be written in terms of spin-half variables $\vec{S}_{\vec{r}}=\vec{\sigma}_{\vec{r}} / 2$ (where $\vec{\sigma}_{\vec{r}}$ are Pauli matrices) representing the total spin of each triangle:

$$
\mathcal{H}_{\mathrm{YL}}=\frac{1}{2} \sum_{\left\langle\vec{r} \vec{r}^{\prime}\right\rangle_{\lambda}} K \tau_{\vec{r}}^{\lambda} \tau_{\overrightarrow{r^{\prime}}}^{\lambda} \vec{\sigma}_{\vec{r}} \cdot \vec{\sigma}_{\vec{r}^{\prime}}-\sum_{\vec{r}} \vec{B} \cdot \vec{S}_{\vec{r}}
$$

Here, $\vec{B}$ is the external magnetic field, the first sum is over all nearest-neighbor links $\left\langle\vec{r} \vec{r}^{\prime}\right\rangle_{\lambda}$ on the honeycomb lattice, $\lambda=x, y, z$ denotes the orientation of the nearest neighbor link connecting the $A$-sublattice site $\vec{r}$ to the $B$-sublattice site $\vec{r}^{\prime}$ of the honeycomb lattice, $\tau_{\vec{r}}^{z}$ is the orbital quantum number introduced above, and $\tau_{\vec{r}}^{x, y}$ are the other two Pauli matrices in this orbital Hilbert space.

Since a nonmagnetic impurity (missing spin) on a triangle $\vec{r}$ of the original frustrated magnet on the decorated honeycomb lattice leads to a nondegenerate (inert) singlet state for this triangle, nonmagnetic impurities in the original model on the decorated honeycomb lattice must be modeled by omitting the corresponding $S_{\vec{r}}$ and $\tau_{\vec{r}}$ in $\mathcal{H}_{\mathrm{YL}}$. Thus, such nonmagnetic impurities give rise to vacancy disorder in the effective model on the honeycomb lattice. In addition, bond disorder in the coupling $K$ of $\mathcal{H}_{\mathrm{YL}}$ arises as a consequence of quenched disorder in the strength of the multispin interaction, so long as the intratriangle exchange $J$ remains the largest coupling in the system. In what follows, we will assume this to be true and analyze the effects of bond and site disorder in $\mathcal{H}_{\mathrm{YL}}$.

We begin by noting that the Hamiltonian $\mathcal{H}_{\mathrm{YL}}$ admits an exact solution $[36,37]$ in terms of a Majorana representation [46,47]: $\sigma_{\vec{r}}^{z}=-i c_{\vec{r}}^{x} c_{\vec{r}}^{y}, \tau_{\vec{r}}^{z}=-i b_{\vec{r}}^{x} b_{\vec{r}}^{y}$, and cyclic permutations thereof, where $c_{\vec{r}}^{\lambda}$ and $b_{\vec{r}}^{\lambda}$ are Majorana (real) fermion operators. In the physical Hilbert space, which is characterized by the local constraint $D_{\vec{r}} \equiv c_{\vec{r}}^{x} c_{\vec{r}}^{y} c_{\vec{r}}^{z} b_{\vec{r}}^{x} b_{\vec{r}}^{y} b_{\vec{r}}^{z}=i$, we have the identity $\sigma_{\vec{r}}^{\alpha} \tau_{\vec{r}}^{\beta}=i c_{\vec{r}}^{\alpha} b_{\vec{r}}^{\beta}$. Defining $\mathbb{Z}_{2}$ gauge fields $u_{\vec{r} r^{\prime}} \equiv b_{\vec{r}}^{\lambda} b_{\vec{r}^{\prime}}^{\lambda}$ on $\lambda$ links, the problem reduces in these variables to three flavors of $c$ fermions hopping on the honeycomb lattice while coupled to a common $\mathbb{Z}_{2}$ gauge field $u$ that has no quantum dynamics:

$$
\mathcal{H}_{\mathrm{YL}}=\frac{K}{4} \sum_{\alpha=x, y, z} \sum_{\overrightarrow{\vec{r}}{ }^{\prime}} i u_{\vec{r} \vec{r}^{\prime}} c_{\vec{r}}^{\alpha} c_{\vec{r}^{\prime}}^{\alpha}+\frac{h}{2} \sum_{\vec{r}} i c_{\vec{r}}^{x} c_{\vec{r}}^{y}
$$

where $\vec{B}=h \hat{z}$. In other words, the model reduces to a collection of free fermion problems, one for each static configuration of $\mathbb{Z}_{2}$ fluxes threading faces of the lattice. In consequence, the temperature-dependent susceptibility for the spin model, including the effects of exchange disorder and vacancies, can be determined from the density of states of an associated free fermion system.

The following dictionary summarizes this correspondence: Physical properties of the SU(2) symmetric spin 
model are controlled by the behaviour of a triplet of Majorana fermions hopping on the honeycomb lattice. The hopping matrix $\mathbf{K}$ has matrix elements $\pm i K_{\vec{r}_{A} \vec{r}_{B}} u_{\vec{r}_{A} \vec{r}_{B}}$ with $u$ taking values corresponding to the ground state flux sector (here $\vec{r}_{A}$ and $\vec{r}_{B}$ are the $A$ and $B$ sublattice sites connected by the corresponding link of the honeycomb lattice). For the pure system or with weak exchange disorder, this is the zero-flux sector. In the diluted case, each vacancy binds a $\pi$ flux in the ground-state flux sector, at least at low dilution $[6,7,37,48-51]$.

We introduce canonical (complex) fermions, defined as $f_{\vec{r}}= \pm\left(c_{\vec{r}}^{x}+i c_{\vec{r}}^{y}\right) / 2$, with the plus (minus) sign on $A(B)$ sublattice sites. The $f$-fermion Hamiltonian is then a tightbinding model with hopping matrix $\mathbf{K}$, single-particle eigenenergies $\epsilon$, and density of states $\rho(\epsilon)$. The magnetic field $h$ acts as a chemical potential for the $f$ fermions, since $S_{\vec{r}}^{z}=-i c_{\vec{r}}^{x} c_{\vec{r}}^{y}=1 / 2-f_{\vec{r}}^{\dagger} f_{\vec{r}}$. Therefore, the magnetic susceptibility of the spin model is equivalent to the compressibility of the $f$-fermion system, determined entirely by $\rho(\epsilon)$. This conclusion is unaffected by the constraint on $D_{\vec{r}}$ [37,48-50] (see Supplemental Material [52] for details). For $h=0$, particle-hole (chiral) symmetry of $\mathbf{K}$ on the bipartite honeycomb lattice ensure that $\rho(\epsilon)$ is an even function of $\epsilon$ and the corresponding chemical potential lies at $\epsilon=0$. The low temperature susceptibility is therefore controlled by the form of the fermion density of states $\rho(\epsilon)$ near $\epsilon=0$ in the presence of disorder.

From universality arguments and previous studies of the bipartite random hopping problem [9,53-57], we know that exchange randomness generates low-energy states that lead to a characteristic divergence of $\rho(\epsilon)$ as $\epsilon \rightarrow 0$. The form of this low-energy density of states has a renormalization group interpretation in terms an infinite-disorder fixedpoint $[21,22,52,55]$. Site dilution is also expected to produce a continuous contribution $\rho_{\text {reg }}(\epsilon)$ similarly divergent for $\epsilon \rightarrow 0$, albeit with a different functional form [6,7,58-61]. In addition, since the tight-binding model for diluted graphene has been shown to host a nonzero density of topologically protected zero modes [58] (whose existence is insensitive to bond disorder), one expects that dilution will generically lead to a nonzero density of zero modes in the present case too, since the $\pi$ flux bound to each vacancy in the ground state flux sector is just another form of bond disorder.

Incorporating the existence of a thermodynamically significant density of zero modes into our parametrization of $\rho$, we write $\rho(\epsilon)=\rho_{0} \delta(\epsilon)+\rho_{\text {reg }}(\epsilon)$. Measuring the fermion energy and temperature in units of the disorderaveraged exchange coupling $K_{\mathrm{av}}$ and using the parametrizations $\Gamma_{\epsilon} \equiv \log _{10}\left(K_{\mathrm{av}} / \epsilon\right)$ and $\Gamma_{T} \equiv \log _{10}\left(K_{\mathrm{av}} / T\right)$, we express the integrated density of states as a function of $\Gamma_{\epsilon}$ via $N\left(\Gamma_{\epsilon}\right)=\int_{-\epsilon}^{\epsilon} d \epsilon^{\prime} \rho_{\text {reg }}\left(\epsilon^{\prime}\right)$.

In the generic bond-disordered case, $\rho_{0}=0$, and we expect $N\left(\Gamma_{T}\right)=T^{\alpha(T)}$, with $\alpha(T)$ vanishing slowly with $T$.
This, and the precise form of $\alpha(T)$ at low $T$ follows from the fact that $|d N(\Gamma) / d \Gamma|$ for large $\Gamma$ has the modified GadeWegner form [53,54] $a \exp \left(-b \Gamma^{1 / x}\right)$ with $x=3 / 2[55,56]$ in the corresponding random hopping problem. For the drifting susceptibility exponent $\alpha(T)$, this implies vanishing $\alpha(T) \propto 1 / \Gamma_{T}^{1 / 3}$ in the low- $T$ limit [62]. Thus, bond disorder on its own gives a low temperature susceptibility of purely random-singlet form [55].

In the site-diluted case, we compute $\rho(\epsilon)$ using the arbitrary-precision methods developed in Ref. [58]. These methods allow us to extend to much lower energies previous studies of site dilution [6,7], and to unambiguously distinguish exact zero modes from very low-energy contributions to $\rho_{\text {reg }}(\epsilon)$. The form of $\mathbf{K}$ that enter these numerical calculations differs from the tight-binding models for disordered graphene studied in Refs. [58-61] due to the $\pi$ flux bound to each vacancy. Representative numerical results are summarized in Fig. 2, and backed up by extensive additional numerical results in the Supplemental Material [52].

Interestingly, we find that the main features of the lowenergy density of states in this site-diluted case are not sensitive to the presence of these $\mathbb{Z}_{2}$ fluxes. Indeed, we find $N\left(\Gamma_{T}\right)=T^{\alpha(T)}$, with $\alpha(T)$ vanishing slowly but nonuniversally as $T \rightarrow 0$, with a characteristic crossover behavior seen earlier in diluted graphene [58]: $\alpha(T) \sim$ $y\left(n_{v}\right) \log \left(\Gamma_{T}\right) / \Gamma_{T}$ at not-too-low temperature, which crosses over to $\alpha(T) \sim 1 / \Gamma_{T}^{1 / 3}$ below the crossover temperature $T_{c} \sim K_{\mathrm{av}} 10^{-\Gamma_{c}\left(n_{v}\right)}$. Both $y\left(n_{v}\right)$ and $T_{c}\left(n_{v}\right)$ decrease quite rapidly with decreasing concentration $n_{v}$ of vacancies, implying a correspondingly stronger singularity in the random singlet form of the susceptibility for lower values of vacancy density. As all the key features of the low-energy physics of the bond-disordered case are already present here, the presence of additional bond disorder in such diluted samples does not lead to any qualitative changes in this low temperature physics.

Returning to the frustrated magnet $\mathcal{H}_{\mathrm{YL}}$ and relating the susceptibility of the spin liquid to the compressibility of the canonical fermions, we obtain for $T \ll K_{\mathrm{av}}$

$$
\chi(T)=\frac{\rho_{0}}{4 T}+\frac{N\left(\Gamma_{T}\right)}{4 T} .
$$

Disorder-induced low-energy fermion states with density $\rho_{\text {reg }}(\epsilon)$ are responsible for the second contribution to $\chi(T)$, with its characteristic random-singlet divergence as $T \rightarrow 0$. For both bond disorder [55] and vacancy disorder [7], this random-singlet form of $\rho_{\text {reg }}$ admits qualitatively similar interpretations in terms of pairs of would-be zero modes that mix very weakly with each other, giving rise to lowenergy tails in $\rho_{\text {reg }}(\epsilon)$. Additionally, in the site-diluted case, exact zero modes give rise to the first term, a Curie tail in the susceptibility with Curie coefficient $\mathcal{C}=\rho_{0} / 4$. 

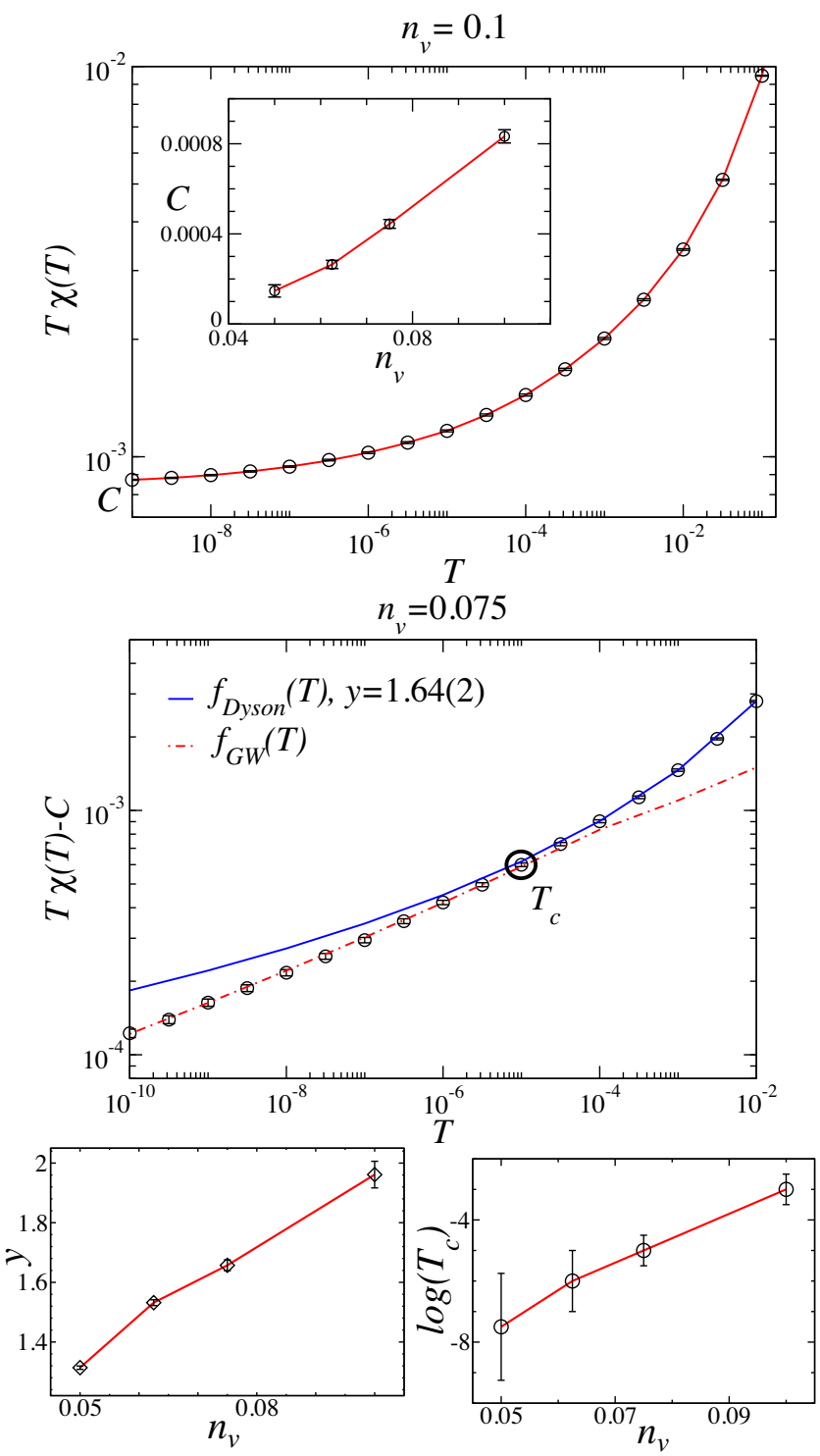

FIG. 2. Top: $T \chi(T)$ for a system with density of vacancies $n_{v}=10 \%$; note the saturation at low temperature, corresponding to a nonzero Curie constant $\mathcal{C}$. Inset shows the $n_{v}$ dependence of $\mathcal{C}$. Middle: $T \chi(T)-\mathcal{C}$ at $n_{v}=7.5 \%$ shows a clear crossover at a temperature scale $T_{c}$ from the functional form $f_{\text {Dyson }}=$ $a /\left[\log ^{y}\left(K_{\mathrm{av}} / T\right)\right]$ at intermediate temperature to $f_{\mathrm{GW}}=$ $b \exp \left[-c \log ^{2 / 3}\left(K_{\mathrm{av}} / T\right)\right]$ at the lowest temperatures. Bottom: Dependence on $n_{v}$ of $y$ (left) and $T_{c}$ (right).

The Curie tail dominates over the random-singlet term at the lowest temperatures, and reflects the presence of free emergent moments whose spatial form factor is given by the local density of these exact zero modes; $\mathcal{C}$ can be extracted directly from the low-temperature limit of $T \chi(T)$ (Fig. 2).

These emergent moments extend over many unit cells, and are very different in spatial profile from isolated free spins. To see this, we first note that $\rho_{0}$ obtained with $\pi$ flux attached to each vacancy (as is appropriate at low

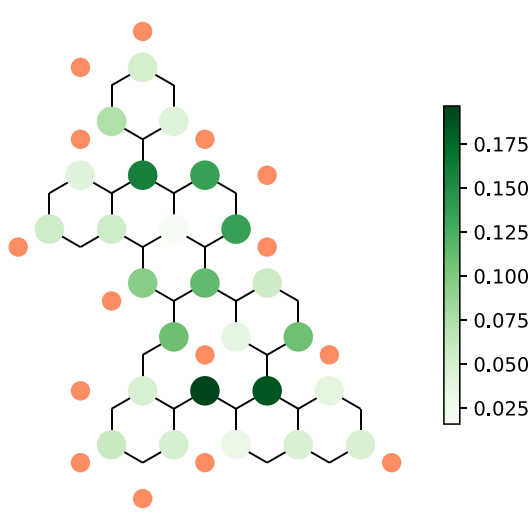

FIG. 3. Vacancy disorder in the honeycomb-lattice effective model $\mathcal{H}_{\text {YL }}$ gives rise to emergent free moments, arising from topologically protected zero modes of the Majorana fermion excitations. Such modes are localized within "R -type regions" [58] of the lattice with local sublattice imbalance, with several modes typically coexisting in a single region [63]. An infinitesimal $z$-magnetic field at $T=0$ immediately polarizes such emergent moments, yielding a ground-state spin texture $\left\langle\sigma_{r}^{z}\right\rangle$. The figure displays a simple example corresponding to two such zero modes in a $\mathcal{R}$-type region seeded by the fifteen vacancies (denoted by orange circles). Bonds $K$ are drawn from box distribution with $20 \%$ width around mean. Bonds outside of this region and sites with $\left\langle\sigma_{r}^{z}\right\rangle=0$ are not shown, but included in calculation. All sites with $\left\langle\sigma_{r}^{z}\right\rangle \neq 0$ are denoted by color-coded circles and belong to one sublattice.

temperature for $\mathcal{H}_{\mathrm{YL}}$ ) is very close to the corresponding density obtained earlier [58] in the site-diluted tightbinding model for graphene (which differs by the absence of these bound $\pi$ fluxes) for dilutions $n_{v} \lesssim 0.1$. Viewing the $\pi$ fluxes as a form of bond disorder (in the signs of the hopping amplitudes), this strongly suggests that most of the modes contributing to $\rho_{0}$ in the present case are topologically protected, i.e., insensitive to bond disorder. This is also consistent with earlier work [58] on diluted graphene, which attributes the dominant component of $\rho_{0}$ to topologically protected zero modes located in so-called $\mathcal{R}$-type regions of the lattice (see Fig. 3), characterized by local sublattice imbalance and a specific structure of their boundary; this boundary structure protects them from mixing with other modes elsewhere in the lattice [58]. From a recent analysis of their random geometry [63], which finds that their typical size scales approximately as $\xi \sim n_{v}^{-5}$ at small $n_{v}$, we conclude that the ground-state spin textures associated with these emergent free moments are spread over correspondingly large areas.

Turning to Kitaev's original honeycomb model, we note that the spectrum of Majorana excitations is identical to the one analyzed here. The random-singlet contribution arising from $\rho_{\text {reg }}$ at small nonzero $|\epsilon|$ now depends on the mean square amplitude of the corresponding wave functions at sites adjacent to vacancies [7]. This wave function dependence is expected to lead to some modification of the 
temperature-dependent exponent $\alpha(T)$, without affecting the random-singlet form of this contribution, since wave functions at nonzero $\epsilon$ in the random hopping problem are expected to be localized. By an analogous argument in the site-diluted case, we also expect that the zero-mode density $\rho_{0}$ gives rise to a Curie constant $\mathcal{C}=\mathcal{A} \rho_{0}$, where the proportionality constant $\mathcal{A}$ represents the mean square zero-mode amplitudes at sites adjacent to vacancies. Crucially, $\mathcal{A}\left(n_{v}\right)$ is expected to be nonzero in the thermodynamic limit since the on-shell zero-energy Green's function is generically localized [63] at any nonzero dilution $n_{v}>0$.

Given the rather comprehensive theoretical understanding achieved here, and given the existence of Kitaev materials [51] with significant vacancy disorder, particularly Kitaev materials that exhibit some signatures of vacancy-induced random-singlet behavior [64], the question of experimental implications naturally becomes important. First of all, the insensitivity of our results with regard to the choice of flux sector implies that experimentally relevant perturbations whose only effect is to favor a different ground-state flux configuration will not affect the basic phenomenology. When perturbations endow the fluxes with dynamics, we expect our results to remain valid above a crossover temperature set by the strength of such perturbations. Even with these perturbations present, our results correctly predict the integrated spectral weight of triplet excitations below this scale, i.e., the combined contribution of the Curie tail and the random-singlet excitations below this scale, along with the corresponding signature in the specific heat. How this integrated weight is then redistributed in specific experimentally relevant cases is thus the key question that needs to be addressed theoretically on a case-by-case basis, with the form of the specific heat below this scale providing experimental guidance. For some peturbations, this may even yield as yet unexplored variants of cooperative physics of the lowenergy emergent degrees of freedom in a disordered, strongly interacting topological magnet.

We thank F. Evers, J. Knolle, O. I. Motrunich, N. Perkins, and M. Vojta for engaging discussions, and R. Bhola and S. Biswas for assistance with Fig. 3. K. D. gratefully acknowledges a fruitful collaboration [63] with R. Bhola, S. Biswas, and M. Islam on the random geometry of disorder-robust zero modes. J. T. C. thanks the Department of Theoretical Physics (DTP) of the Tata Institute of Fundamental Research (TIFR) for hospitality during a visit in which this collaboration was initiated, and gratefully acknowledges travel support from the OxfordIndia Theoretical Physics Network for this visit; his work is also supported by EPSRC Grant No. EP/S020527/1. This work was in part supported by the Deutsche Forschungsgemeinschaft under grants SFB 1143 (project-id 247310070) and the cluster of excellence ct.qmat (EXC 2147, project-id 390858490). S. S. and K. D. gratefully acknowledge use of computational resources of DTP (TIFR) and the International Center for Theoretical Sciences TIFR (ICTS-TIFR) for all the computational work described here. Several of the computational results obtained by S. S. first appeared in his Ph.D thesis (2015) submitted to the TIFR Deemed University. S. S. was funded by a graduate fellowship of the TIFR during a major part of his work, by a postdoctoral fellowship of ICTS-TIFR in the final stages of his work, and by SERB, DST India via a start-up research grant (SRG/2020/ 001525) during the drafting and finalization of this manuscript. K. D. was supported at the TIFR by DAE (India), and partially by SERB, DST India via a J.C. Bose Fellowship (JCB/2020/000047) and by the InfosysChandrasekharan Random Geometry Center (TIFR).

[1] L. Balents, Spin liquids in frustrated magnets, Nature (London) 464, 199 (2010).

[2] J. Knolle and R. Moessner, A field guide to spin liquids, Annu. Rev. Condens. Matter Phys. 10, 451 (2019).

[3] H. Takagi, T. Takayama, G. Jackeli, G. Khaliullin, and S. E. Nagler, Concept and realization of Kitaev quantum spin liquids, Nat. Rev. Phys. 1, 264 (2019).

[4] F. Alet, A. M. Walczak, and M. P. A. Fisher, Exotic quantum phases and phase transitions in correlated matter, Physica (Amsterdam) 369A, 122 (2006).

[5] C. Castelnovo, R. Moessner, and S. L. Sondhi, Magnetic monopoles in spin ice, Nature (London) 451, 42 (2008).

[6] A. J. Willans, J. T. Chalker, and R. Moessner, Disorder in a Quantum Spin Liquid: Flux Binding and Local Moment Formation, Phys. Rev. Lett. 104, 237203 (2010).

[7] A. J. Willans, J. T. Chalker, and R. Moessner, Site dilution in the Kitaev honeycomb model, Phys. Rev. B 84, 115146 (2011).

[8] K. Gregor and O. I. Motrunich, Nonmagnetic impurities in a $S=1 / 2$ frustrated triangular antiferromagnet: Broadening of ${ }^{13} \mathrm{C}$ NMR lines in $\kappa-(\mathrm{ET})_{2} \mathrm{Cu}_{2}(\mathrm{CN})_{3}$, Phys. Rev. B 79, 024421 (2009).

[9] J. Knolle, R. Moessner, and N. B. Perkins, Bond-Disordered Spin Liquid and the Honeycomb Iridate $\mathrm{H}_{3} \mathrm{LiIr}_{2} \mathrm{O}_{6}$ : Abundant Low-Energy Density of States from Random Majorana Hopping, Phys. Rev. Lett. 122, 047202 (2019).

[10] T. H. Han, M. R. Norman, J.-J. Wen, J. A. RodriguezRivera, J. S. Helton, C. Broholm, and Y. S. Lee, Correlated impurities and intrinsic spin-liquid physics in the kagome material herbertsmithite, Phys. Rev. B 94, 060409(R) (2016).

[11] K. Kitagawa, T. Takayama, Y. Matsumoto, A. Kato, R. Takano, Y. Kishimoto, S. Bette, R. Dinnebier, G. Jackeli, and H. Takagi, A spin-orbital entangled quantum liquid on the honeycomb lattice, Nature (London) 554, 341 (2018).

[12] S-K. Ma, C. Dasgupta, and C-K. Hu, Random Antiferromagnetic Chain, Phys. Rev. Lett. 43, 1434 (1979); Erratum, Phys. Rev. Lett. 43, 1899 (1979).

[13] C. Dasgupta and S-K. Ma, Low-temperature properties of the random Heisenberg antiferromagnetic chain, Phys. Rev. B 22, 1305 (1980). 
[14] H. M. Bozler, C. M. Gould, and W. G. Clark, Crossover Behavior of a Random-Exchange Heisenberg Antiferromagnetic Chain at Ultralow Temperatures, Phys. Rev. Lett. 45, 1303 (1980).

[15] D. S. Fisher, Random antiferromagnetic quantum spin chains, Phys. Rev. B 50, 3799 (1994).

[16] D. S. Fisher, Random transverse-field Ising chains, Phys. Rev. B 51, 6411 (1995).

[17] R. A. Hyman and K. Yang, Impurity Driven Phase Transition in the Antiferromagnetic Spin-1 Chain, Phys. Rev. Lett. 78, 1783 (1997).

[18] G. Refael, S. Kehrein, and D. S. Fisher, Spin reduction transition in spin-3/2 random Heisenberg chains, Phys. Rev. B 66, 060402(R) (2002).

[19] K. Damle, Griffiths effects in random Heisenberg antiferromagnetic $S=1$ chains, Phys. Rev. B 66, 104425 (2002).

[20] K. Damle and D. A. Huse, Permutation-Symmetric Multicritical Points in Random Antiferromagnetic Spin Chains, Phys. Rev. Lett. 89, 277203 (2002).

[21] K. Damle, O. I. Motrunich, and D. A. Huse, Dynamics and Transport in Random Antiferromagnetic Spin Chains, Phys. Rev. Lett. 84, 3434 (2000).

[22] O. I. Motrunich, K. Damle, and D. A. Huse, Dynamics and transport in random quantum systems governed by strongrandomness fixed points, Phys. Rev. B 63, 134424 (2001).

[23] F. J. Dyson, The dynamics of a disordered linear chain, Phys. Rev. 92, 1331 (1953).

[24] T. P. Eggarter and R. Riedinger, Singular behavior of tightbinding chains with off-diagonal disorder, Phys. Rev. B 18, 569 (1978).

[25] M. A. Paalanen, A. E. Ruckenstein, and G. A. Thomas, Spins in Si: P Close to the Metal-Insulator Transition, Phys. Rev. Lett. 54, 1295 (1985).

[26] Z.-Z. Gan and P. A. Lee, Nuclear-spin relaxation near the metal insulator transition, Phys. Rev. B 33, 3595(R) (1986).

[27] R. N. Bhatt and P. A. Lee, Scaling Studies of Highly Disordered Spin-1/2 Antiferromagnetic Systems, Phys. Rev. Lett. 48, 344 (1982).

[28] O. I. Motrunich, S-C Mau, D. A. Huse, and D. S. Fisher, Infinite-randomness quantum Ising critical fixed points, Phys. Rev. B 61, 1160 (2000).

[29] R. R. P. Singh, Valence Bond Glass Phase in Dilute Kagome Antiferromagnets, Phys. Rev. Lett. 104, 177203 (2010).

[30] I. Kimchi, A. Nahum, and T. Senthil, Valence Bonds in Random Quantum Magnets: Theory and Application to $\mathrm{YbMgGaO}_{4}$, Phys. Rev. X 8, 031028 (2018).

[31] L. Liu, H. Shao, Y.-C. Lin, W. Guo, and A. W. Sandvik, Random-Singlet Phase in Disordered Two-Dimensional Quantum Magnets, Phys. Rev. X 8, 041040 (2018).

[32] I. Kimchi, J. P. Sheckelton, T. M. McQueen, and P. A. Lee, Scaling and data collapse from local moments in frustrated disordered quantum spin systems, Nat. Commun. 9, 4367 (2018).

[33] J. P. Sheckelton, J. R. Neilson, D. G. Soltan, and T. M. McQueen, Possible valence-bond condensation in the frustrated cluster magnet $\mathrm{LiZn}_{2} \mathrm{Mo}_{3} \mathrm{O}_{8}$, Nat. Mater. 11, 493 (2012).

[34] J. P. Sheckelton, F. R. Foronda, L. Pan, C. Moir, R. D. McDonald, T. Lancaster, P. J. Baker, N. P. Armitage,
T. Imai, S. J. Blundell, and T. M. McQueen, Local magnetism and spin correlations in the geometrically frustrated cluster magnet $\mathrm{LiZn}_{2} \mathrm{Mo}_{3} \mathrm{O}_{8}$, Phys. Rev. B 89, 064407 (2014).

[35] K. T. Law and P. A. Lee, $1 \mathrm{~T}-\mathrm{TaS}_{2}$ as a quantum spin liquid, Proc. Natl. Acad. Sci. U.S.A. 114, 6996 (2017).

[36] H. Yao and D.-H. Lee, Fermionic Magnons, Non-Abelian Spinons, and the Spin Quantum Hall Effect from an Exactly Solvable Spin-1/2 Kitaev Model with SU(2) Symmetry, Phys. Rev. Lett. 107, 087205 (2011).

[37] A. Kitaev, Anyons in an exactly solved model and beyond, Ann. Phys. (Amsterdam) 321, 2 (2006).

[38] P. Schiffer and I. Daruka, Two-population model for anomalous low-temperature magnetism in geometrically frustrated magnets, Phys. Rev. B 56, 13712 (1997).

[39] R. Moessner and A. J. Berlinsky, Magnetic Susceptibility of Diluted Pyrochlore and $\mathrm{SrCr}_{9-9 x} \mathrm{Ga}_{3+9 x} \mathrm{O}_{19}$ Antiferromagnets, Phys. Rev. Lett. 83, 3293 (1999).

[40] A. Sen, K. Damle, and R. Moessner, Vacancy-induced spin textures and their interactions in a classical spin liquid, Phys. Rev. B 86, 205134 (2012).

[41] A. Sen, K. Damle, and R. Moessner, Fractional Spin Textures in the Frustrated Magnet $\mathrm{SrCr}_{9 p} \mathrm{Ga}_{12-9 p} \mathrm{O}_{19}$, Phys. Rev. Lett. 106, 127203 (2011).

[42] J. Rehn, A. Sen, A. Andreanov, K. Damle, R. Moessner, and A. Scardicchio, Random Coulomb antiferromagnets: From diluted spin liquids to Euclidean random matrices, Phys. Rev. B 92, 085144 (2015).

[43] P. Patil, F. Alet, S. Capponi, and K. Damle, Quantum halforphans in kagomeé magnets, Phys. Rev. Research 2, 043425 (2020).

[44] R. R. Biswas, L. Fu, C. R. Laumann, and S. Sachdev, $\mathrm{SU}(2)$-invariant spin liquids on the triangular lattice with spinful Majorana excitations, Phys. Rev. B 83, 245131 (2011).

[45] H.-H. Lai and O. I. Motrunich, SU(2)-invariant Majorana spin liquid with stable parton Fermi surfaces in an exactly solvable model, Phys. Rev. B 84, 085141 (2011).

[46] A. M. Tsvelik, New Fermionic Description of Quantum Spin Liquid State, Phys. Rev. Lett. 69, 2142 (1992).

[47] B. S. Shastry and D. Sen, Majorana fermion representation for an antiferromagnetic spin-1/2 chain, Phys. Rev. B 55, 2988 (1997).

[48] E. H. Lieb, Flux Phase of the Half-Filled Band, Phys. Rev. Lett. 73, 2158 (1994).

[49] F. L. Pedrocchi, S. Chesi, and D. Loss, Physical solutions of the Kitaev honeycomb model, Phys. Rev. B 84, 165414 (2011).

[50] F. Zschocke and M. Vojta, Physical states and finite-size effects in Kitaev's honeycomb model: Bond disorder, spin excitations, and NMR line shape, Phys. Rev. B 92, 014403 (2015).

[51] W-H. Kao, J. Knolle, G. B. Halász, R. Moessner, and N. B. Perkins, Vacancy-Induced Low-Energy Density of States in the Kitaev Spin Liquid, Phys. Rev. X 11, 011034 (2021).

[52] See Supplemental Material at http://link.aps.org/ supplemental/10.1103/PhysRevLett.127.127201 for additional computational details and supporting evidence. 
[53] R. Gade, Anderson localization for sublattice models, Nucl. Phys. B398, 499 (1993).

[54] R. Gade and F. Wegner, The $\mathrm{n}=0$ replica limit of $\mathrm{U}(\mathrm{n})$ and U(n)/SO(n) models, Nucl. Phys. B360, 213 (1991).

[55] O. Motrunich, K. Damle, and D. A. Huse, Particle-hole symmetric localization in two dimensions, Phys. Rev. B 65, 064206 (2002).

[56] C. Mudry, S. Ryu, and A. Furusaki, Density of states for the $\pi$-flux state with bipartite real random hopping only: A weak disorder approach, Phys. Rev. B 67, 064202 (2003).

[57] O. Motrunich, K. Damle, and D. A. Huse, Griffiths effects and quantum critical points in dirty superconductors without spin-rotation invariance: One-dimensional examples, Phys. Rev. B 63, 224204 (2001).

[58] S. Sanyal, K. Damle, and O. I. Motrunich, Vacancy-Induced Low-Energy States in Undoped Graphene, Phys. Rev. Lett. 117, 116806 (2016).

[59] V. Hafner, J. Schindler, N. Weik, T. Mayer, S. Balakrishnan, R. Narayanan, S. Bera, and F. Evers, Density of States in Graphene with Vacancies: Midgap Power Law and
Frozen Multifractality, Phys. Rev. Lett. 113, 186802 (2014).

[60] P. M. Ostrovsky, I. V. Protopopov, E. J. König, I. V. Gornyi, A. D. Mirlin, and M. A. Skvortsov, Density of States in a Two-Dimensional Chiral Metal with Vacancies, Phys. Rev. Lett. 113, 186803 (2014).

[61] N. Weik, J. Schindler, S. Bera, G. C. Solomon, and F. Evers, Graphene with vacancies: Supernumerary zero modes, Phys. Rev. B 94, 064204 (2016).

[62] Very similar results have been obtained more recently for the spectrum averaged over flux sectors [9], consistent with the expectation [53-56] that the low-energy behaviour would be independent of flux sector.

[63] R. Bhola, S. Biswas, M. Islam, and K. Damle, DulmageMendelsohn percolation: Geometry of maximally-packed dimer models and topologically-protected zero modes on diluted bipartite lattices, arXiv:2007.04974v5.

[64] S.-H. Baek, H. W. Yeo, S.-H. Do, K.-Y. Choi, L. Janssen, M. Vojta, and B. Buchner, Observation of a random singlet state in a diluted Kitaev honeycomb material, Phys. Rev. B 102, 094407 (2020). 